\title{
Enhancing images of facial expressions
}

\author{
PHILIP J. BENSON \\ University of Oxford, Oxford, England \\ RUTH CAMPBELL and TANYA HARRIS \\ University of London, London, England \\ MARK G. FRANK \\ Rutgers University, New Brunswick, New Jersey \\ and \\ MARTIN J. TOVÉE \\ University of Newcastle-upon-Tyne, Newcastle, England
}

\begin{abstract}
Facial images can be enhanced by application of an algorithm - the caricature algorithm - that systematically manipulates their distinctiveness (Benson \& Perrett, 1991c; Brennan, 1985). In this study, we first produced a composite facial image from natural images of the six facial expressions of fear, sadness, surprise, happiness, disgust, and anger shown on a number of different individual faces (Ekman \& Friesen, 1975). We then caricatured the composite images with respect to a neutral (resting) expression. Experiment 1 showed that rated strength of the target expression was directly related to the degree of enhancement for all the expressions. Experiment 2, which used a free rating procedure, found that, although caricature enhanced the strength of the target expression (more extreme ratings), it did not necessarily enhance its purity, inasmuch as the attributes of nontarget expressions were also enhanced. Naming of prototypes, of original exemplar images, and of caricatures was explored in Experiment 3 and followed the pattern suggested by the free rating conditions of Experiment 2, with no overall naming advantage to caricatures under these conditions. Overall, the experiments suggested that computational methods of compositing and caricature can be usefully applied to facial images of expression. Their utility in enhancing the distinctiveness of the expression depends on the purity of expression in the source image.
\end{abstract}

Since Darwin (1872/1965), the ways in which facial expressions may relate to felt emotions, intentions, and motivational states have intrigued researchers into the nature of the perception and classification of expression. The dominant view is that there is reasonable reliability across different human groups in interpreting these facial actions and that these reflect a finite number of underlying categories - either emotional (see Ekman, 1989, 1993; Ekman, Sorenson, \& Friesen, 1969; Izard, 1971, 1994) or dispositional (Fridlund, 1991). Although there is disagreement concerning the structure of these categories and their variability across cultures (Ekman, 1994; Izard, 1994; Russell, $1980,1991,1994)$, there is consensus that, at the very least, facial expressions show bounded visual family resemblances (Ekman, 1982, 1993). If emotional expressions

P.J.B. acknowledges the support of the U.K. Medical Research Council, the Oxford McDonnell-Pew Centre for Cognitive Neuroscience, the Oxford MRC Centre in Brain and Behaviour, and the James S. McDonnell Foundation. We thank Niall Scott for assisting with data collection for Experiments 1 and 2. Our thanks go also to Paul Ekman and two anonymous referees for their helpful comments. Correspondence concerning this article should be addressed to P. J. Benson, University Laboratory of Physiology, University of Oxford, Parks Road, Oxford OX1 3PT, England (e-mail: philip.benson@physiol.ox.ac.uk). are perceived as being discrete categories, images of emotional expression should show the psychological characteristics of categorical perception. Three published studies suggest that this is indeed the case. Etcoff and Magee (1992) used line drawings derived computationally from standard images of facial expression (Ekman, 1982). They systematically interpolated between each expression-face, constructing a series of images for each expression continuum (i.e., $100 \%$ happy, $90 \%$ happy- $10 \%$ sad, $80 \%$ happy $-20 \%$ sad, ... 100\% sad). Subjects perceived this continuum discontinuously. Both Calder, Young, Perrett, Etcoff, and Rowland (1996) and de Gelder, Teunisse, and Benson (1997) extended these findings to photographic images of facial expressions, using a computational morphing technique developed and described by Benson $(1992,1994 a)$, and confirmed the line-drawings findings. The present study extends the computer-based analysis and synthesis of images of facial expression, by exploring a phenomenon related to the categorization of facial expression.

\section{Caricature}

Caricatures of facial identity can be produced by an algorithm that systematically enhances the distinctiveness between face images. Using line drawings of faces, Brennan (1985) derived feature coordinates across a series and 
then averaged them to obtain normative values (the prototypical face for that population). The difference between the specific exemplar values and the prototype values provides a measure of the distinctiveness of that face with respect to a population of faces. New face images can then be produced that either increase (caricature) or decrease (anticaricature) these differences systematically. When the distinctiveness of the face was increased (caricature), the resemblance to the individual was often enhanced, leading to faster identification; when it was decreased (anticaricature), it took longer to identify (Rhodes, Brennan, \& Carey, 1987). ${ }^{1}$ Some caricatures may even be judged to be more like the person than is a veridical image (the superportrait effect; Benson, 1998; Benson \& Perrett, 1994; Mauro \& Kubovy, 1992; Rhodes, 1996; Rhodes \& Tremawan, 1994). Benson (1998; Benson \& Perrett, 1991b, 1991c) further explored the effects of identity enhancement by extending the algorithm from line drawings to facial photographs. Similar effects were obtained, although not always to the same extent as with line drawings. Linedrawn exemplars tend to be poor likenesses, because they lack important skin texture information, and so can offer more scope for enhancement than do tonal images based on photographs (which tend to be seen as good likenesses in the first place).

\section{Averaging and Caricaturing for Expression}

Brennan's (1985) algorithm for caricature can be used to enhance the differences between a reference image, and a target image or set of images. In the experiments reported here, we performed two operations on photographic images of facial expression from a public domain published source (Ekman \& Friesen, 1975). In the first operation, we averaged face feature values for each expression across the different individual face images producing that expression (six different faces). In this way, we obtained composite expression images for each of the six facial categories of expression. We performed the same operation for resting (neutral) faces from the same set. We then obtained the face-feature difference values between each expression composite and the neutral composite in order to obtain an expression difference measure. By increasing the value of the difference measure, the distinctiveness of the expression image was enhanced to form a caricature. By decreasing this value, a less distinctive (anticaricature) image was produced. A series of such images was produced for each expression.

\section{Using an Expression Composite}

In a recently reported study, Calder, Young, Rowland, and Perrett (1997) followed a procedure similar to that outlined above to explore questions similar to those posed here. However, the source image for their caricature manipulation was the image of a particular individual, rather than an image composited across a number of different individuals displaying that expression. We preferred to use the composite image for the following reasons. First, compositing with the Benson and Perrett (1993) face-feature landmark technique had not been reported for facial expression and offered the opportunity to assess the robustness of the technique to capture expression (Benson, 1995). Second, individual image differences are reduced in the compositing procedure; any idiosyncrasies of the individual face and of its expression are minimized (see Galton, 1907). Thus, if, for instance, judges are biased to give different ratings of expression, depending on the age or gender of the face they are inspecting, these will be reduced when the image, a composite, appears to be of indeterminate gender or age. Third, the compositing technique means that findings may generalize to other images and to other individual faces better than they would if a single individual were the source of the image.

\section{The Feature-Based Algorithm}

There are a number of ways to manipulate facial images computationally, including facial expressions (see, e.g., Bartlett et al., 1996). In our studies, a face-feature landmark algorithm was used. A number of points on each and every face image were located by hand. These delineated the shape and disposition of the face features and their outlines, and the vectors derived from these values formed the basis for manipulation. Unlike a pixel-by-pixel (imagebased) technique, this obviates the need for refined face normalization. It also ensures that the image is clear and not blurred. Further justification for this technique and examples of it are described in Benson and Perrett (1991a, 1993 ) and Benson (1994b, 1995). More details of the technique are also outlined below (see the Method section).

The question of interest in this study is primarily empirical: Does caricature work for facial expression images, as it can for identity? What level of enhancement works best and for which expressions? The issue is also of theoretical interest: Image-based approaches to face processing (e.g., O'Toole, Deffenbacher, Valentin, \& Abdi, 1994; Valentin, Abdi, O'Toole, \& Cottrell, 1994) suggest that the distinctiveness of facial images may be best captured in multidimensional vector space. Following this kind of approach, a face made more distinctive by caricature may be easier to categorize, because its vectors will tend to lie further from those of other face examples; perceptual distinctiveness is captured in the mathematical distribution of the vectors of the face set. However, we cannot know in advance where the boundaries of such distortions may lie. For instance, a face caricatured for happiness may tip over into an expression such as anger. It could, more generally, be argued that, because caricature is a motivated distortion of a good or a prototypical example of a facial category, caricature effects-should they occur-may offer strong support for a categorical theory of the classification of emotional expression in faces. We shall return to this point in the General Discussion section, with experimental evidence to hand.

In the first experiment reported here, different levels of enhancement (positive and negative) of photographic images of faces were investigated in a rating task (goodness of category fit). That is, the rated strength of the image for 
that expression was measured. In the second experiment, an open rating task was administered, in which the subjects rated each of the facial images for its rated proportion of each of the six posited expressions - that is, the rated purity of the image was measured. Finally, in Experiment 3 , judges were shown prototypes, exemplars, and caricatures for speeded naming.

\section{Image Production: Compositing the Source (Prototype) Image for Each Expression}

The source images for these studies were the facial images of expression published in Unmasking the Face (Ekman \& Friesen, 1975). These were composed of a series of full-face photo portraits of eight different individuals, all identically lit and disposed, which can be classified as portraying one or another of the six facial expressions of happiness, surprise, disgust, anger, fear, and sadness, as well as a neutral resting face. The basis for the production of the facial poses was, for two of the posers, an early version of the Facial Action Coding System (FACS) facial muscle action scoring system, developed more fully by Ekman and colleagues (Ekman \& Friesen, 1978; Ekman, Friesen, \& Ellsworth, 1972). This system details the precise muscle actions required to produce agreed-on best depictions of the six universal facial expressions. The other sitters in this series were, however, informed simply to pose the required expression. The photographs varied somewhat in the intensity of the expression portrayed, as was confirmed by a full rating study undertaken more recently on these and other images (Ekman, 1982; Ekman \& Friesen, 1978; Ekman et al., 1972). Thus, in selecting the faces for compositing, we make no claim that these were the best and most representative images for that expression, only that the expressions gleaned from this set of individuals were good enough. This was confirmed prior to the experiment by asking three judges to label each of the source images, using one of the six expression labels. This indicated a $100 \%$ agreement between judges' matchings to the label.

Thus, six images for each expression, from six different posers including the two FACS-instructed posers, were flatbed scanned from the published photographs as continuoustone images and processed on a Silicon Graphics workstation, using custom software (Benson \& Perrett, 1991c). A set of 224 landmarks was manually logged by reference to previously established templates (Benson \& Perrett, 1993) on each face. These described the topographic arrangement of key facial features and the outline of the face in the head. Each image was normalized by rotational transformation to the vertical midline and scaled for equal interpupil distance. The average position of each feature across the six images was then obtained by calculating the mean position of each of the respective landmarks of each such normalized face image. After this, digital image warping (Benson \& Perrett, 1991c) was applied to each expression, using the original landmarks and the respective expression-averaged landmarks. The warping algorithm (affine warping) first tessellated the facial image by form- ing triangles between every neighboring landmark point. Each such triangular tile was then distorted into the new, average shape. The same algorithm was then applied to texture and tone within each tile. In this way, the shape and tonal image qualities of each of the six source images for each of the six expressions were averaged to produce a single composite image for each of the six facial expressions and for a neutral expression.

\section{Image Production: The Enhanced Facial Image}

For each composite facial image, the landmark values were compared with those of the neutral face and enhanced systematically, following Brennan's (1985) algorithm, which uses the difference between the source image and a baseline control (in this case, the neutral expression composite; see Benson \& Perrett, 1991c). Four levels of enhancement were used to create four variants on the prototype composite. These were $-100 \%$ (equivalent in configuration to the neutral face) $,-50 \%,+50 \%$, and $+100 \%$. Thus, in the $+100 \%$ caricature, each feature distance (prototype minus neutral) difference was doubled. Negative value enhancements are anticaricatures, positive ones caricatures. The composite prototype value is zero. Prototyped and 100\% enhanced stimuli are shown in Figure 1.

\section{EXPERIMENT 1}

This study ${ }^{2}$ examined within-expression category ratings by 13 naive adult subjects. If caricature operates systematically for expression, positive caricatures should be rated more highly for that expression, and negative ones less highly.

\section{Method}

Subjects. Seven male and 6 female undergraduates at the University of Newcastle-upon-Tyne, England, participated voluntarily.

Display. When displayed on the Silicon Graphics (Indy R4000) workstation monitor, images subtended a visual angle of about $15^{\circ} \times$ $12^{\circ}$ at a viewing distance of about $60 \mathrm{~cm}$. The name of the relevant expression was shown beneath each image. Under this, a horizontal mouse-controlled graphical slider was presented with an indicated range from 1, very poor, to 11 , very good.

Procedure. The subjects performed the experiment individually. They were instructed to rate each displayed image for the emotion label, using the interactive slider. Once they had been shown how to start each trial and had completed one trial run, they proceeded at their own pace. To start the display, the slider had to be manipulated away from the start position. The observer then pressed the workstation keyboard spacebar to $\log$ the estimate and to cue the next trial. Five repeated blocks were presented, each of which comprised all the experimental images (six expressions, four levels of caricature, prototypes), each shown once and in a random order. Each stimulus was visible until the rating had been completed. Testing lasted about $12 \mathrm{~min}$, with a break between each block

\section{Results}

An analysis of variance (ANOVA) of mean ratings (SPSS MANOVA) was conducted on the means of the ratings across subjects (see Figure 2). There were two factors: level of enhancement (five levels) and expression 

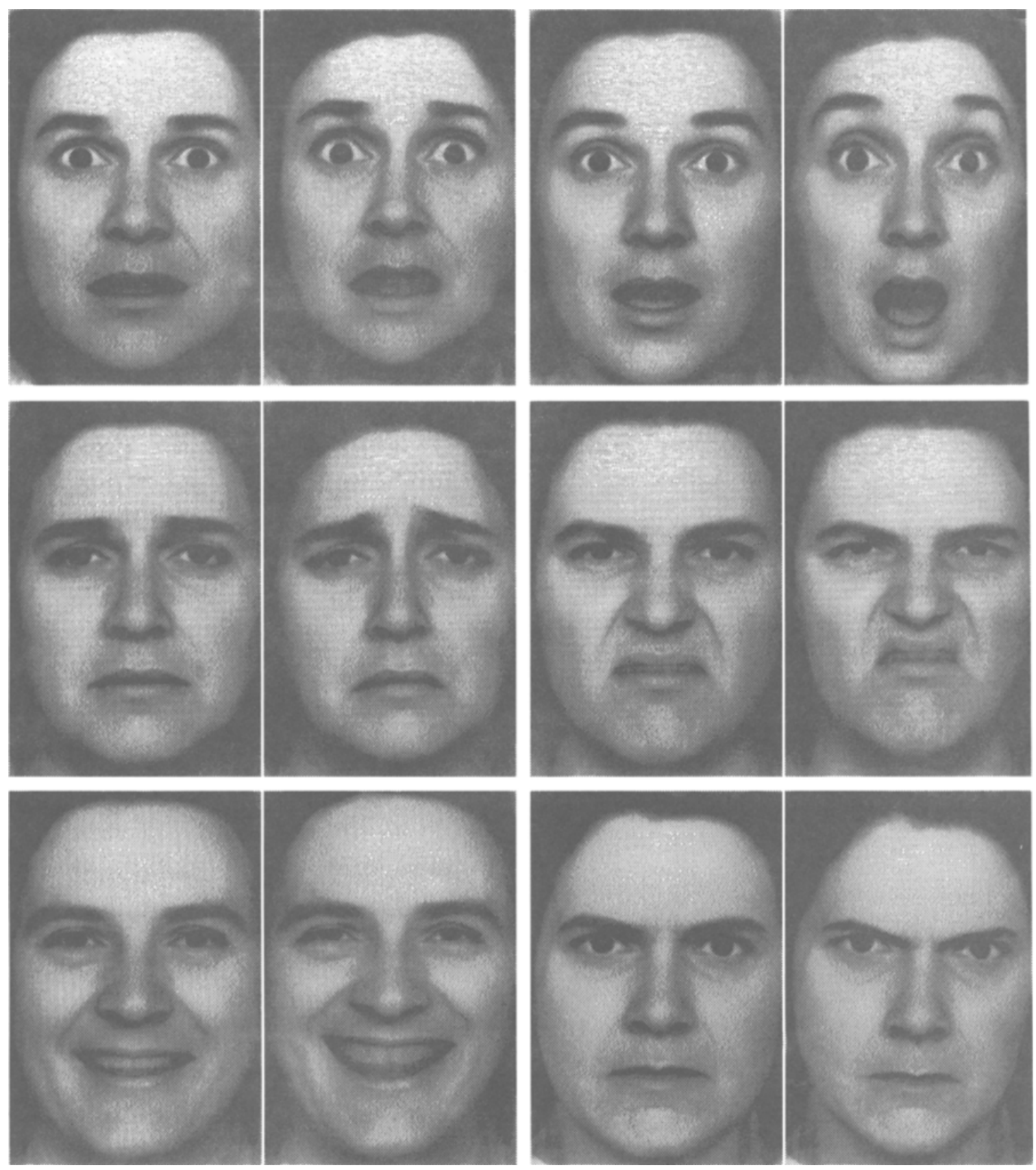

Figure 1. Composite prototype and caricature (enhanced) stimuli used in the studies. Images of six different individuals posing each of the six target expressions (Ekman \& Friesen, 1975) were averaged across individuals (Benson \& Perrett, 1993) to generate composite prototype stimuli for each expression. These were modified (Benson \& Perrett, 1991c) to generate caricature-enhanced stimuli. Each pair of images shows the (left) prototype and (right) enhanced stimulus. Enhanced stimuli are twice $(+100 \%)$ the difference between the neutral prototype and the prototype condition. From top-left, left-toright: fear, surprise, sadness, disgust, happiness, and anger.

(six types). This confirmed a significant effect of enhancement $[F(4,48)=171.92, p<.0001]$, as well as a main effect of expression $[F(60,5)=4.04, p<.01]$. The interaction term (univariate) was also significant $[F(240,20)=$ $3.98, p<.001]$. Planned comparisons confirmed that the effect of degree of caricature was in the following order:
$100 \%$ anticaricature $\ll 50 \%$ anticaricature $<$ normal $=$ $50 \%$ caricature $<100 \%$ caricature (where $\ll=p<.001$ and $<=p<.01$ ). That is, the major effect of enhancement was in the anticaricature range.

A separate ANOVA contrasted normal images and $100 \%$ caricatures. This was a two-factor within-subjects 


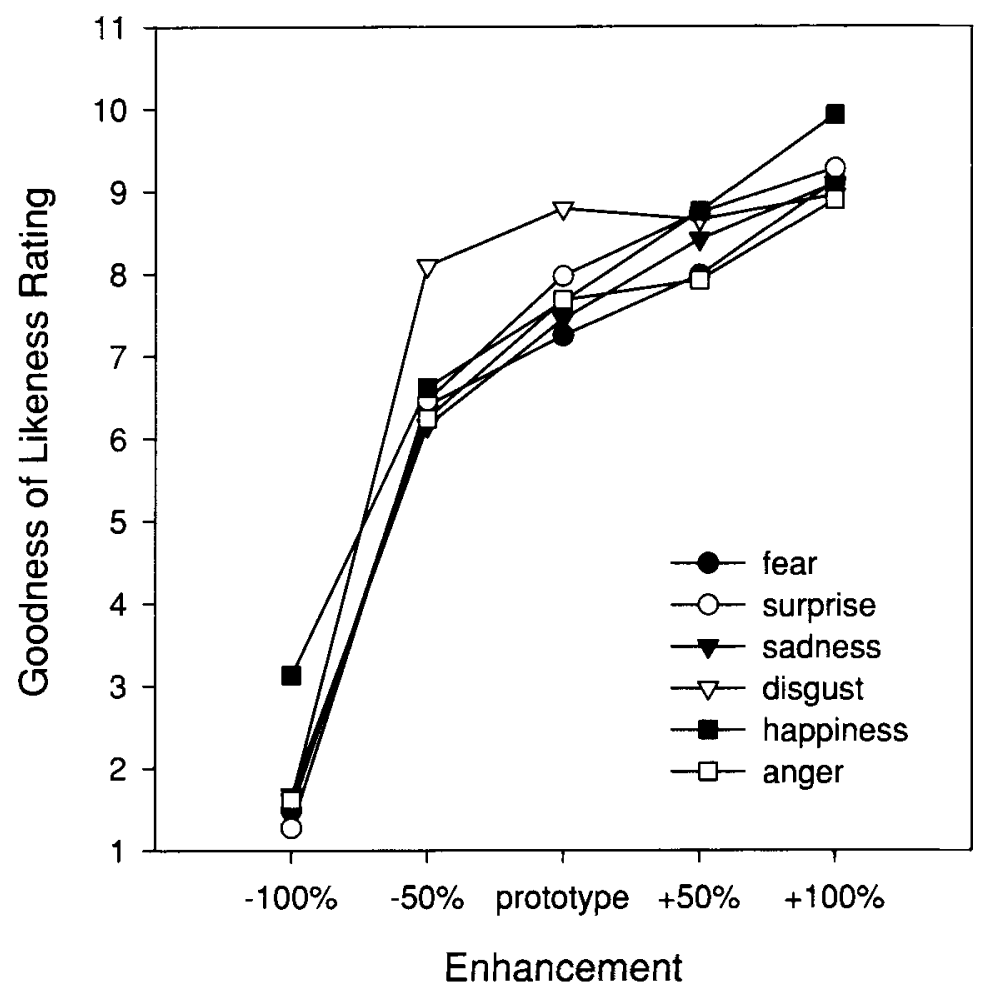

Figure 2. Mean ratings of positive and negative enhancement. The subjects rated each presented image for emotion content on an 11-point scale (1, very poor, to 11, very good). Stimuli were presented at five levels of emotion.

ANOVA, with expression as one factor (six levels) and caricature (present or absent) as the other. Under these restricted conditions, there was no significant main effect of expression $[F(60,5)=1.86]$, but the effect of caricature remained significant $[F(12,1)=48.32, p<.001]$, as did the interaction term $[F(60,5)=3.76, p<.005]$. It was shown with $t$ tests on individual expressions that all the expressions generated significant caricature effects, except for disgust $(t=0.35)$. Figure 3 summarizes these observations.

\section{Discussion}

This experiment shows that linear enhancement of the distinctiveness of facial expressions by an established computational algorithm makes those images better likenesses of that expression when enhancement is positive and worse likenesses when enhancement is negative. The ratings also suggest that the composited image was itself a good example of the rated expression, typically gaining ratings of 7 or more on the 11 -point scale.

The function that relates degree of caricature to rated goodness of expression is not itself linear over the range explored here. The relatively greater effect of negative enhancement than of positive enhancement may reflect a number of possibilities. One is that there may be a category boundary between a perceived neutral expression and the target expression in the $-100 \%$ to $-50 \%$ range. There is no suggestion that $100 \%$ caricature tips the expression into an- other category. Even though the positive effects seem quite small, as compared with the negative ones, positive caricatures $(100 \%)$ were rated higher than the composite prototype faces for each of the tested expressions, with one exception: Disgust was sensitive to the enhancement algorithm but did not show a caricature advantage in this study. It reached maximal goodness at the prototype stage.

Another point worth noting is that the anticaricatured faces generated positive values on all expressions, but especially on the happy expression. This in turn suggests that the neutral face prototype had a mildly happy aspect. In a separate study (data from Experiment 2, but not reported here), this was confirmed: Typically, the neutral face was more likely to be rated as being happy than as being anything else. ${ }^{3}$

\section{EXPERIMENT 2}

Although Experiment I showed that the fit of a single expression label to an image was sensitive to caricature, it did not test whether this was specific to that expression. Expression may be gauged in two ways: Its strength or its purity may be the basis for rating. A face may be judged to be quite happy because it is on a perceived continuum between happy and neutral or because it is perceived to be on a continuum between happy and (for example) surprise. In this experiment, the subjects were asked to rate each image in terms of all the constituent emotions expressed. This is also 


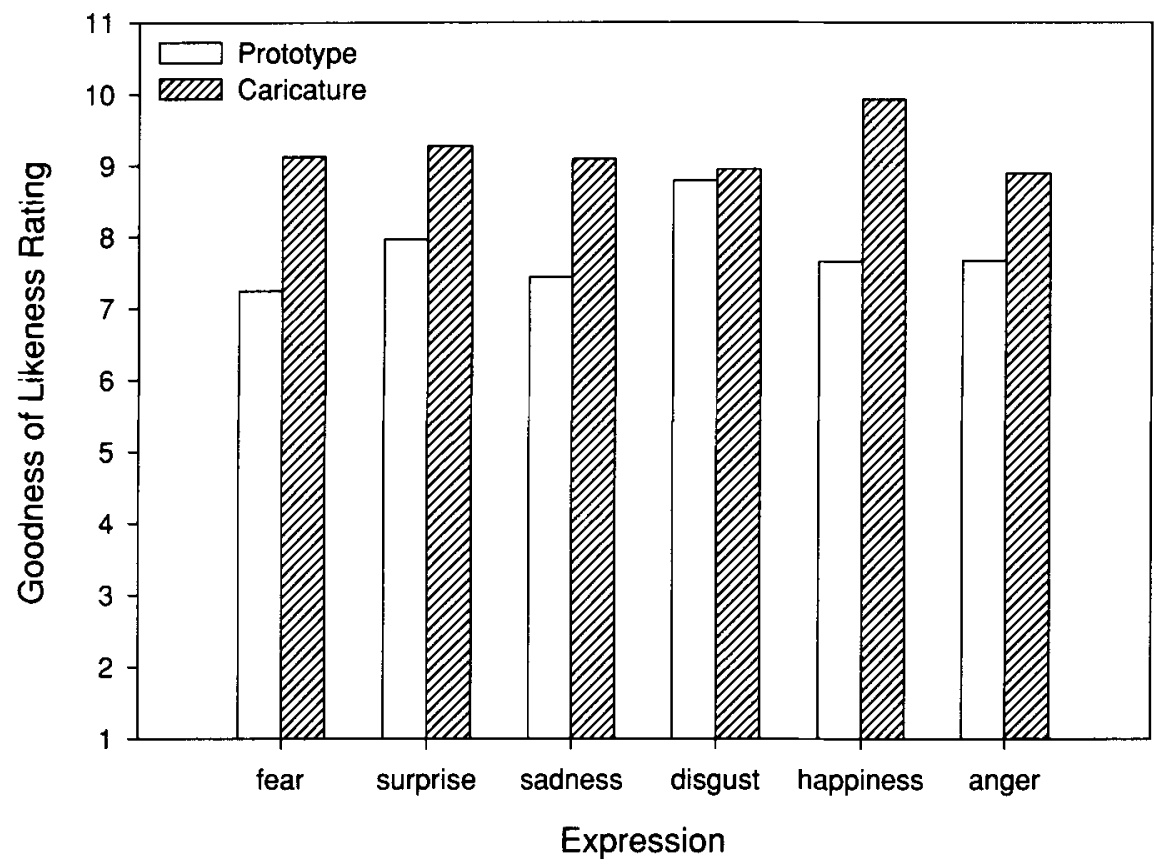

Figure 3. Experiment 1: Effects of expression enhancement. Direct comparison of subjects' mean ratings obtained for the prototype and caricatured (enhanced) stimuli on an 11-point scale (1, very poor, to 11, very good). For all six basic expressions (fear, surprise, sadness, disgust, happiness, and anger) caricature enhancement of the composite prototype increased image ratings.

important in order to establish more clearly the extent to which the use of the face-feature algorithm to derive the composite face for each emotion worked. The question of interest here is, Does caricature enhance the perceived strength of an expression, with respect to the neutral baseline, or its purity - that is, with respect to the target expression compared with all the others-or both? Only ratings for prototypes and $100 \%$ caricatures are reported in this study.

\section{Method}

Subjects. There were 8 male and 7 female undergraduate naive volunteers. They were from the same pool as those in the previous experiment.

Procedure. The same procedure was followed as that for Experiment 1 , using the same images (six expressions at each of five levels and a neutral face). However, this time, the display did not carry the expression name; rather, six horizontal sliders were shown below the image. These were separately labeled for each of the expressions (happiness through to sadness) and had a percentage scale on each, from (marked) $0 \%$ (not present) to $100 \%$ (maximally present). All the sliders were set to 0 at the start of each trial, and at least one of them had to be moved, if only to be returned to 0 during the trial. Once more, five blocks comprising one random presentation of each of the images were given. Pressing the spacebar logged the rating and cued the next trial. The stimulus was visible until all ratings had been completed and the spacebar pressed. Testing lasted about $45 \mathrm{~min}$, with a break between blocks.

\section{Results}

This report details the analyses for $100 \%$ caricatures and prototypes. Means were derived across all the subjects for expression ratings for every expression category and are shown in Figure 4. ANOVAs were performed on subject means for each expression separately. The factors (within-subjects) were enhancement (prototype or caricature) and expression (six levels). The results are summarized in Table 1.

Caricature increased ratings overall for all the expressions except fear. The ratings also show the specificity of the enhancement effect in terms of the interaction between the effect of expression and caricature. This was significant for surprise, sadness, happiness, and anger but not for fear or disgust.

\section{Discussion}

This experiment extended the findings of Experiment 1 . Caricatures were rated more highly than prototypes overall and significantly so for every emotion except fear. That is, caricatures produce stronger emotional expressions than do the composite prototype image from which they were drawn. Were effects of enhancement specific to the target expression? Figure 4 shows somewhat greater enhancement for the target expression than for others, while the significant interaction between expression and caricature for all the expressions except fear and disgust (Table 1) could be taken to suggest that caricature also increased the ratings of the target expression, as compared with the others. However, this was by no means uniform across the expressions and was absent for some.

Fear. The prototype fear face was also highly rated for surprise. Caricature enhanced both of these ratings and did not reduce this difference significantly. 

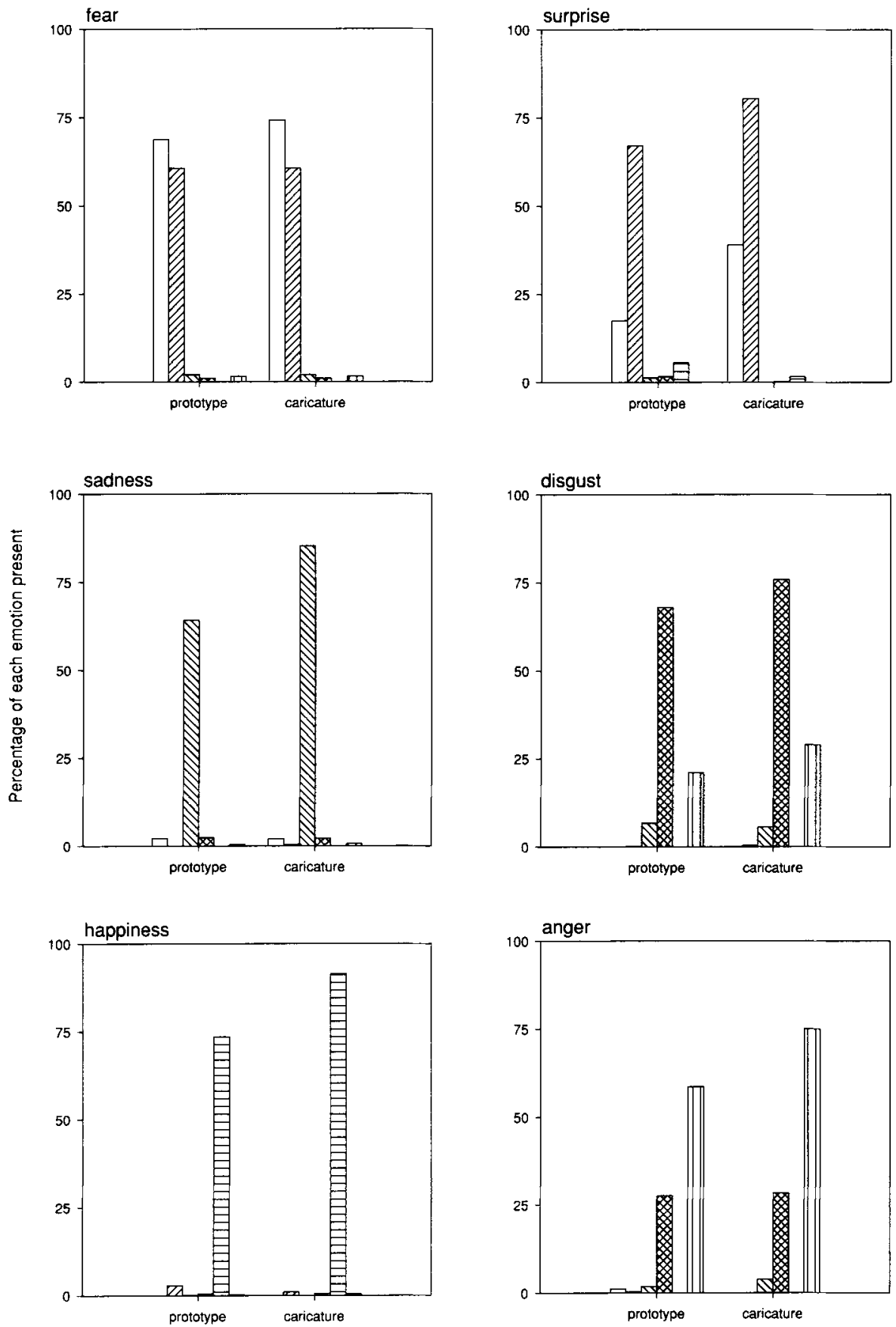

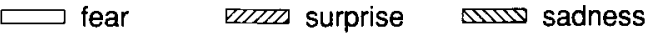

$$
\begin{aligned}
& \text { disgust } \sqsubset \text { happiness } \square \text { anger }
\end{aligned}
$$

Figure 4. Experiment 2. Mean rated expression for each display. Mean proportion of each emotion judged present in the composite prototype and caricatured facial expression. Only happiness and sadness were judged to be pure expressions. 
Table 1

Experiment 2: ANOVA SummaryMode of Representation and Rating Analyses

\begin{tabular}{lcccl}
\hline Expression & $F(14,1)$ Caricature & $F(70,5)$ Expression & $F(70,5)$ Interaction & \multicolumn{1}{c}{ Comparison } \\
\hline Fear & $1.00, \mathrm{n} . \mathrm{s}$. & $95.50, p<.001$ & $1.00, \mathrm{n} . \mathrm{s}$. & fear $>$ surprise $>$ all others \\
Surprise & $18.90, p<.001$ & $140.62, p<.001$ & $14.70, p<.001$ & surprise $>$ fear $>$ all others \\
Sadness & $39.30, p<.001$ & $473.62, p<.001$ & $37.80, p<.001$ & sadness $>$ all others \\
Disgust & $7.11, p<.02$ & $94.67, p<.001$ & $2.26, p=.058$ & disgust $>$ anger $>$ all others \\
Happiness & $35.68, p<.001$ & $557.51, p<.001$ & $33.77, p<.001$ & happiness $>$ all others \\
Anger & $33.40, p<.001$ & $68.59, p<.001$ & $9.50, p<.001$ & anger $>$ disgust $>$ all others \\
\hline
\end{tabular}

Note-Six emotion content ratings for each prototype and caricatured expressions were obtained. The right column indicates interpretation of post hoc Scheffé planned comparisons.

Surprise. The prototype surprise face was also highly rated for fear. Caricature enhanced both of these ratings and did not reduce this difference significantly.

Disgust. The prototype disgust face was also highly rated for anger. If anything, caricature enhanced anger slightly more than it enhanced disgust. Although the anger prototype was also rated quite highly for disgust, caricature did not enhance disgust ratings. If anything, anger was the sole emotion whose purity was enhanced by caricature.

Thus, when the prototype expression was judged to be a pure example of that expression, with low ratings for nontarget expressions, caricature enhanced the ratings of the target expression with little effect on nontargets. However, when the prototype was judged to have greater components of more than one expression, these nontarget expressions were generally also enhanced (and to a similar extent in that target expression). Several independent studies have found similar cross-expression ratings to those reported here (fear labeled surprise, surprise as fear, anger as disgust, and disgust as anger), despite using different images (Izard, 1971; Nummenmaa, 1988; Tomkins \& McCarter, 1964). It is, therefore, likely that these particular confusions reflect some common elements in the facial poses for these expressions. Not all expressions are equally distinct one from the other in terms of the perception, and possibly production, of facial actions.

Taken together, the results of Experiments 1 and 2 show first that a facial image composited across different individuals posing a particular expression can provide a reasonably good prototype for that expression, although happiness and sadness faces were the only ones judged to show that expression and no others. Second, facial expression can be readily enhanced by applying a differencebased algorithm to each expression image with respect to a neutral face. These effects apply to all expressions, although to a different extent for each. When the algorithm is applied, there is a tendency for the caricatured face to be rated more highly for both the target expression and for the second-ranked expression.

\section{EXPERIMENT 3}

Inasmuch as caricature enhances ratings of emotion, can it also enhance naming efficiency? In this experiment, both composite prototypes and their caricatures were pre- sented in the context of other exemplars of the six expressions for speeded naming. If rated strength of expression predicts categorization efficiency, naming should benefit from caricature. However, naming a facial expression requires that it be identified as the target expression in the absence of a cue, such as a category name. Experiment 2 showed that our prototype facial images of fear, surprise, disgust, and anger could carry high ratings for nontarget expressions too. These displays are, therefore, likely to activate not only the target expression but also the highly rated nontarget. In particular, we might expect that those expressions would be hard to name. Because caricature enhances strength but not necessarily purity of expression (Experiment 2), its effect on speed of naming is not predictable. In addition, this study explored naming speed for individual face exemplars of expression (the images from which the composite was drawn).

\section{Method}

Subjects. The observers in this study were 38 adult volunteers, between 22 and 40 years of age. They included students and staff at Goldsmiths College. None had performed in Experiments 1 or 2. All spoke English as a first language.

Materials. A PC was used to display the facial images and record naming times and accuracy.

Images. The target images (prototype and caricature) were embedded within a larger series of exemplars for naming in order to achieve reliable and systematic naming performance in an appropriate context. Prototype and $100 \%$ caricature images from the set used in Experiments 1 and 2 were the target images for this procedure ( 12 images). A further 42 images, 7 for each expression, were chosen from Ekman and Friesen's (1975) published images. These were prerated by three judges as being good examples of the target expression. In addition, left-right mirror reversals of every image were added to the data set. Although there are systematic effects of laterality in posed expression (Borod. St. Clair, Koff, \& Alpert, 1990; Campbell, 1978), pilot studies indicated that, in this context, mirroring did not produce systematic changes in the effects of caricature for different expressions. The reason for producing mirror-reversed as well as normal images was to increase the number of stimuli in the series with small variation but without stimulus repetition.

Procedure. The experiment was run in three blocks. The first block had a series of 42 trials using exemplar images only. This was a practice run, and the images in this session were not presented in the experimental trials. This was followed by two runs of 54 trials. Each run sampled from one set of 54 images without replacement (seven exemplars per expression, six expression prototypes, and six $100 \%$ caricatures). When a normally oriented image was presented 
in the first run, the mirror-reversed version was seen in the second run. Each image was displayed in gray scale $(8 \mathrm{bit})$, image size normalized to about $18 \times 28 \mathrm{~cm}$ viewed at $80 \mathrm{~cm}$. For each subject, responses were averaged over the two experimental runs.

The subjects were tested individually, in a quiet testing room. They wore headphones to which a voice microphone was attached. This was connected to a voice detector that had been calibrated before the experiment, so that the subject's voice stopped the timing device. Instructions for the experiment were displayed on the monitor. These were as follows:

You will see a series of faces, each of which displays an emotion. Your task is to name the emotion on the face as quickly and as accurately as possible. The faces will look happy, sad, surprise, angry, fearful or disgusted. You may think that it looks like none of these, in which case you will say "pass."

Following these instructions, which also stressed that the subjects should not cough or hesitate while wearing the voice microphone, the subjects' questions were dealt with. The experimental sequence started with a warning tone (on headphones), followed by a dark screen for $500 \mathrm{msec}$. This was replaced by the face image at the center of the screen for $250 \mathrm{msec}$. This exposure was found to be optimal for eliciting speeded naming with relatively small variance between subjects. A 4-sec response time was allowed between each sequence. The PC logged naming latencies, whereas the spoken response was logged by hand by the experimenter and later transferred to the data file. The subjects were debriefed following the experiment, which lasted about $20 \mathrm{~min}$.

\section{Results}

Mean correct reaction times (RTs) were recorded for each subject, as was the expression given for each response. RTs greater than $3 \mathrm{sec}$ were scored as errors, along with all responses other than the correct expression name.
Figure 5 shows mean naming speed and accuracy for each expression.

Naming speed. RT was analyzed by ANOVA with expression (six levels) and stimulus type (exemplar, prototype, or caricature) as the two within-subjects factors. Both main effects and their interaction were significant. The main effect of expression gave $F(95,170)=6.42, p<$ .001 . Scheffé tests showed that happiness was the expression named most quickly. Responses to anger were slower than those to surprise and sadness. The main effect of stimulus type (exemplar, composite prototype, or caricature) gave $F(2,68)=3.13, p<.05$. Exemplars were named faster than caricatures (Scheffé, $p<.05$, one-tailed), but the difference between caricature and prototype was not significant. The significant interaction term $[F(2,21)=$ $10.34, p<.01]$ was examined further, using separate ANOVAs for each expression. For anger and fear, there was a significant caricature prototype advantage. Exemplars were faster than composite prototypes for sadness, surprise, fear, and anger expressions.

Accuracy. Accuracy was examined similarly. Figure 6 shows the relevant means, with more detailed expression/ name confusion data in Figure 7. In the overall ANOVA in which expression and type were entered as factors, both had a significant effect [expression: $F(170,5)=6.42, p<$ .01 ; condition: $F(68,2)=3.13, p=.05]$. Happiness was the most accurately reported expression; the order of accuracy was happy $\gg$ sadness $=$ surprise $>$ anger $>$ disgust $>$ fear. The main effect of type was confined to caricature, which was significantly more accurate than the

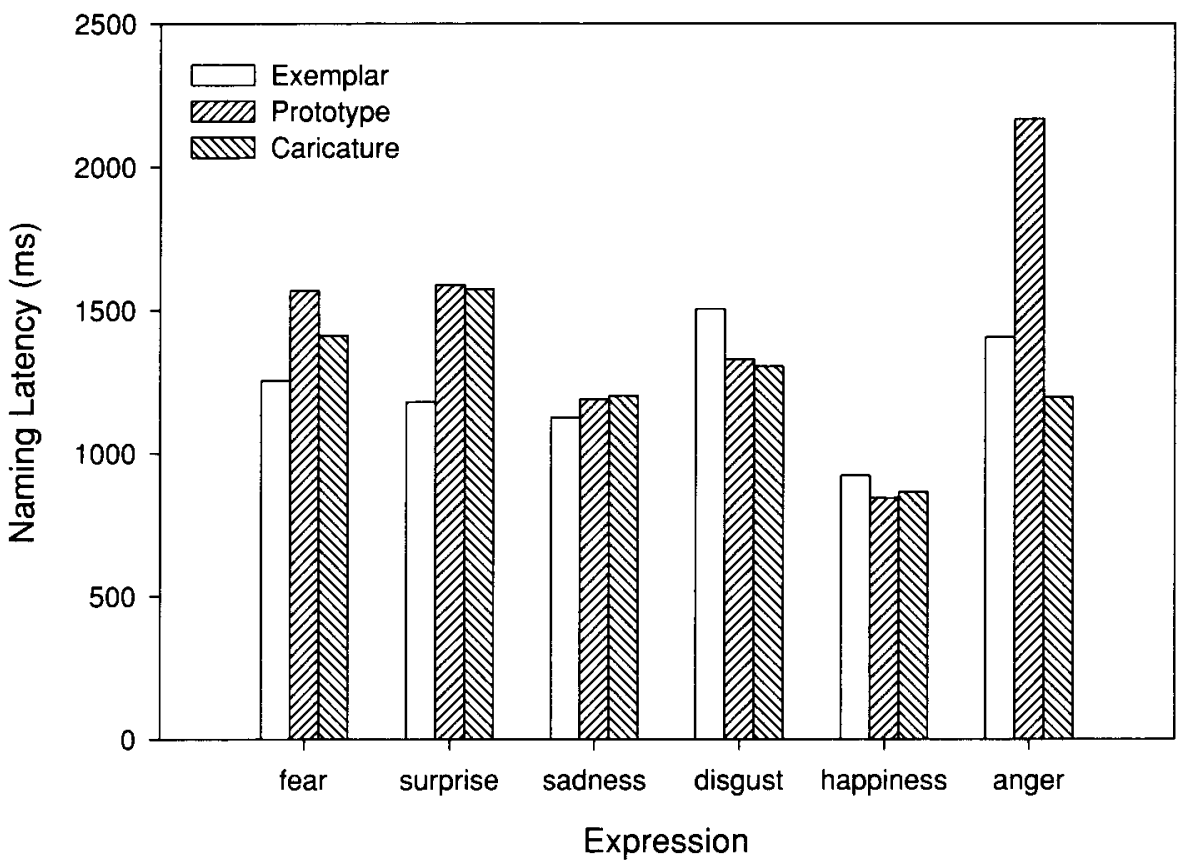

Figure 5. Experiment 3: Mean naming efficiency for each expression. Verbal naming latency recorded during expression identification task for the six basic expressions shown as veridical exemplar, composite prototype, or $+100 \%$ caricature-enhanced prototype. 


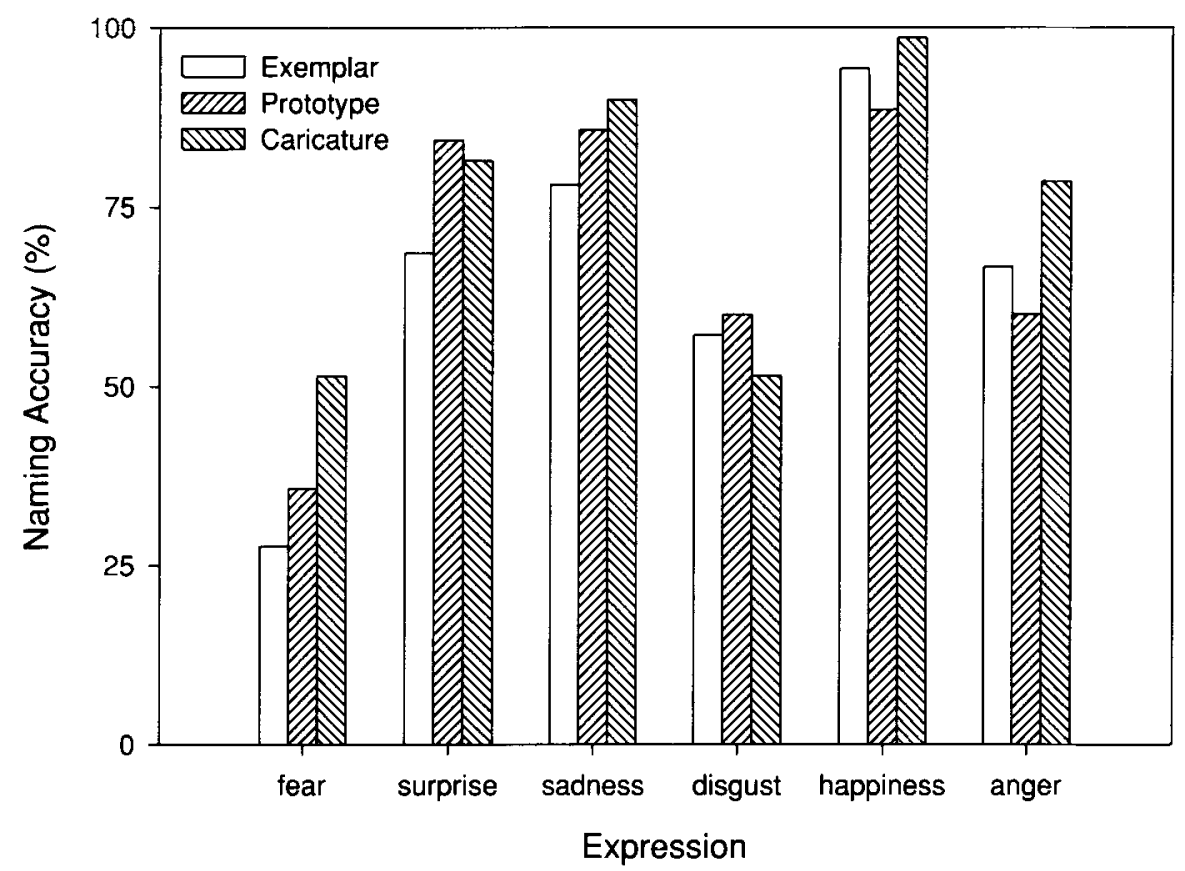

Figure 6. Experiment 3: Correct naming of target expressions. Verbal naming accuracy recorded during expression identification task for the six basic expressions shown as veridical exemplar, prototype, or $+100 \%$ caricature-enhanced prototype.

other two conditions ( $p<.05$, one-tailed). The interaction between these factors was also significant $[F(340,10)=$ $2.21, p=.017]$. Planned comparisons showed that the caricature effect (caricature prototype difference) was only significant for anger.

Expression confusions are shown in Figure 7. This confirms the pattern seen in Experiment 2. In particular, fear was often named as surprise (less so vice versa), and disgust was often named as anger (but not vice versa).

\section{Discussion}

In this experiment, caricature had only minor effects on naming. It failed to enhance naming efficiency generally, although particular expressions, especially anger, were named more quickly and with fewer errors when the caricature rather than the composite prototype (or exemplar) was presented. Happiness was again processed more efficiently than the other expression displays. This general similarity of pattern across the three different experiments will be considered further below, but at this point it is important to note that it suggests that variables underlie the naming response pattern that are similar to those in the ratings tasks. In particular, purity of expression appears to be an important factor: The expressions of fear, disgust, and surprise generated misnamings (Figure 6) that were in line with the ratings found in Experiment 2. Naturally, this appeared more strongly in the accuracy than in the latency results, but even speed of correct naming seems to be sensitive to this factor.

Exemplars and composite prototypes. This study also highlighted further interesting points. The composite prototype face was not necessarily named faster or more accurately than the (mean of) the exemplars from which it derived. Overall, exemplars were correctly named faster than prototypes, although for some expressions (fear, surprise, and sadness), this was offset by more frequent nontarget naming for exemplars than for prototypes.

Although Experiments 1 and 2 suggest that compositing expressions over different individual faces can generate quite good prototypes, this naming study suggests that the exemplars from which the composite derived could, at least sometimes, be better examples of the target expression. Natural variation from person to person for goodness of expression may explain this to some extent (it should be noted that only correct target responses were scored for latency and that most of the expressions showed relatively high rates of misnaming), but a more interesting possibility is that the compositing algorithm fails to capture all the expressive features of the set of exemplar faces. In turn, this implies that a target expression may be expressed and perceived idiosyncratically, rather than in a unique display mode. What might the compositing algorithm miss that is so crucial to interpreting facial expressions? Figure 1 suggests one image property reduced by compositing: Lines generated by skin folds accompanying muscle actions are not particularly pronounced in the composite, other than the nasolabial fold, which was mapped as one of the facefeature landmarks. The feature landmark algorithm captures the position and shape changes of facial features such as eyebrows, eyes, nose, mouth, and chin. If facial fold lines follow these feature movements systematically, they should be captured by the warping algorithm as tone vari- 

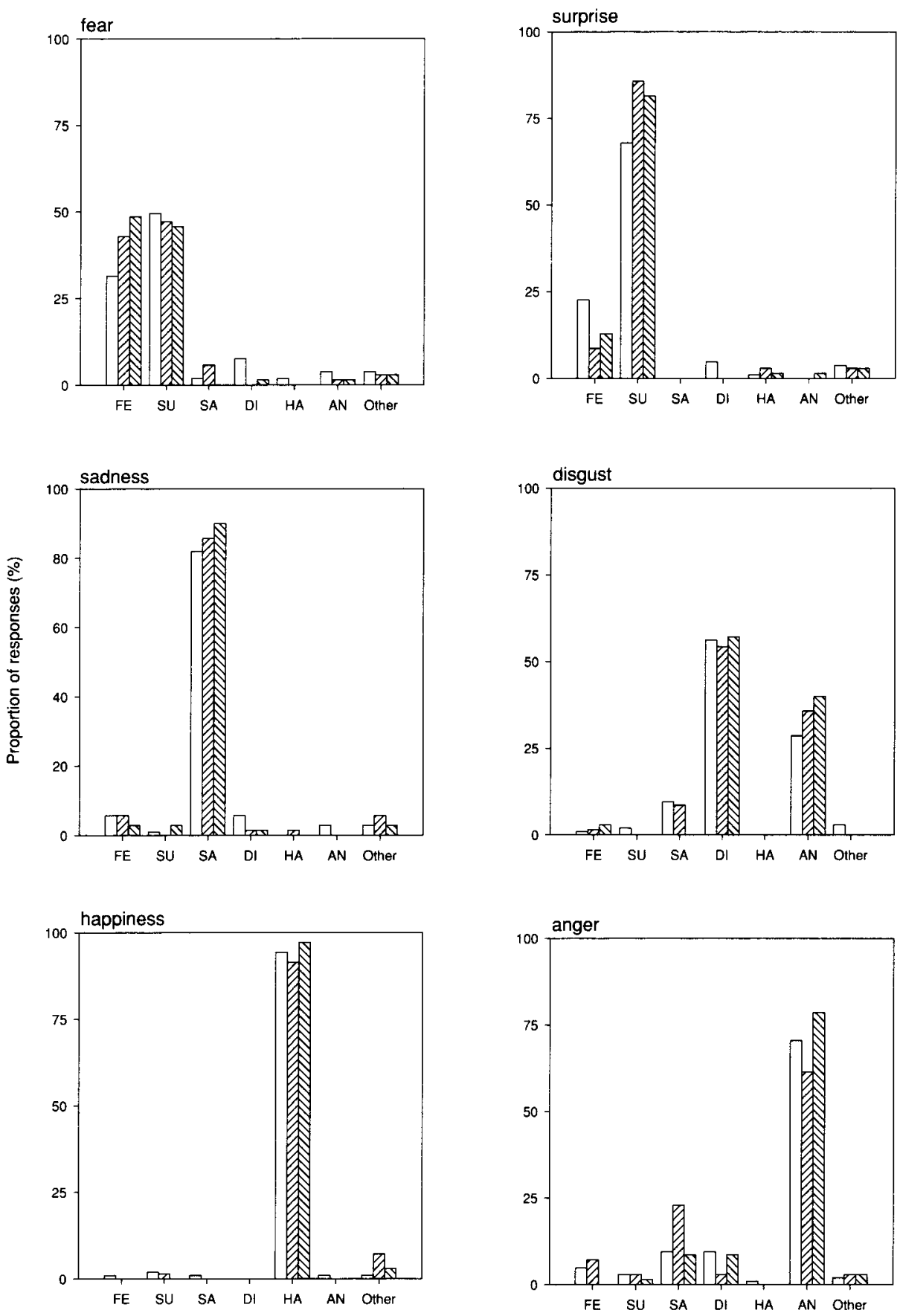

$\square$ exemplar $\mathrm{ETZ}$ prototype $\mathrm{DSU}$ caricature

Figure 7. Experiment 3: Naming confusions. Distribution of names attributed to stimuli during expression labeling task illustrating identification confusions. Confusion errors are in accordance with legal blends (Ekman \& Friesen, 1975; Nummenmaa, 1988) and resemble similar findings in other recognition or rating experiments (Ekman et al., 1969; Tomkins \& McCarter, 1964). Abscissa codes are FEar, SUrprise, SAdness, DIsgust, HAppiness, and ANger. 
ations along the edges of the respective tiles. That they are not suggests that these lines, although expressive of the target, are idiosyncratic in their placement and density and, of course, that such changes in visual texture are important in identifying facial expressions (Benson, in press). However, features such as brow corrugations and orbital eye creases (crow's feet), which were not processed (delineated) in the compositing process, do remain visible, although less pronounced; certain functional nonanatomical features were preserved.

\section{Meta-Analysis and Further Correlations}

Further analysis across the three experiments can help to clarify how caricature works in this context.

Measures of facial action of different expressions in relation to the measures of this study. The excursion of the facial features from a resting (neutral) state varies with the expression portrayed. How might this affect the results reported here? Do such facial energy scores (Benson, 1995) predict the caricature effect (ratings, accuracy, or speed differences between prototype and caricature) for each expression in this study? There is some evidence that, for identity, caricature is most effective for images that are least distinctive (Benson, 1998; Benson \& Perrett, 1991b; Rhodes, Byatt, Tremewan, \& Kennedy, 1997). Extrapolating to the present study, this might predict a negative correlation between energy and caricature: That is, those images with the least energy (closest to neutral) may show the most benefit from caricature. In the following analyses, we explore two measures of facial energy. Both assess the positions of face-feature landmarks for the neutral and for the six target expression-composites. However, the measures themselves are somewhat different. One is essentially qualitative and seeks evidence for specific muscle action units (AUs) contributing to the different displays. The other is a simple measure of the spatial relationships between the face-feature landmarks in the six expressions.

FACS-scored measures of facial energy for our images. FACS (Ekman \& Friesen, 1978) is a descriptive technique for characterizing the production and perception of facial displays of expression primarily, but not exclusively, in terms of the movements and dispositions of the underlying facial muscles. FACS measures of facial feature excursion can be extrapolated onto the facial surface (see, e.g., Bartlett et al., 1996; Essa \& Pentland, 1995; Morishima, 1996). In this study, we do not attempt to convert full FACS scores to feature excursion values, but rather ask how FACS scores for each facial image predict the pattern of results found for composites and caricatures. FACS measures are made by trained researchers, using the coded muscle AU system of Ekman and Friesen (1978).

Table 2 shows the FACS ratings for the composite images used in Experiments 2 and 3. The first question is: Were the composites good examples of the target expression? The answer, generally, is yes. All AUs correspond to those that have been described for these expressions (Ekman \& Friesen, 1978). However, inspection of Table 2 shows that our examples (composite prototypes) of fear and surprise involved almost identical muscle group actions. One distinctive action of a fearful face, the corrugator muscle, could not be discerned in the composite. Was this because the face-feature delineation algorithm was unable to pick up these actions? It seems more likely that

Table 2

FACS Analysis of the Face Images

\begin{tabular}{|c|c|c|c|c|c|c|c|c|c|c|c|c|c|c|c|}
\hline \multirow[b]{2}{*}{ Images } & \multicolumn{15}{|c|}{ FACS Action Unit (AU) } \\
\hline & 1 & 2 & 4 & 5 & 6 & 9 & 10 & 12 & 16 & 17 & 20 & 24 & 25 & 26 & 41 \\
\hline Happy & & & & & + & & & $\mathrm{B}$ & & & & & & + & \\
\hline Happy $100 \%$ & & & & & + & & & D & & & & & & + & \\
\hline Sad & + & & + & & & & & & B & & & & & & + \\
\hline Sad $100 \%$ & + & & + & & & & & & $\mathrm{C}$ & & & & & & + \\
\hline Fear & + & + & & B & & & & & & & & & & + & \\
\hline Fear $100 \%$ & + & + & & D & & & & & & & A & & & + & \\
\hline Surprise & + & + & & $\mathrm{C}$ & & & & & & & & & & + & \\
\hline Surprise $100 \%$ & + & + & & D & & & & & & & & & & + & \\
\hline Anger & & & t & B & & & & & & & & & & & \\
\hline Anger $100 \%$ & & & + & A & & & & & & & & B & & & \\
\hline Disgust & & & & & & + & $\mathrm{C}$ & & & A & & & + & & \\
\hline Disgust $100 \%$ & & & & & & + & $\mathrm{D}$ & & & & & & & + & \\
\hline Neutral & & & & & & & & & & & & & + & & \\
\hline
\end{tabular}

Note-FACS scores (Ekman \& Friesen, 1978) for composite prototype stimuli were assessed by a trained expert (M.G.F.). The codes for the muscle action groups are: 1 , medial portion of frontalis (mid-forehead); 2 , lateral portion of frontalis; 4 , corrugator (lowers eyebrows); 5 , raise eyelids (part of the orbicularis oculi); 6 , lateral portion of obicularis oculi (gives crows feet appearance to eye); 9 , nose wrinkling muscles; 10, levator labii superioris (raises the upper lip); 12, zygomatic major (smile); 16, triangularis (depresses outer corners of lips); 17, mentalis (chin boss; when flexed, pushes lower lip upwards); 20, risorius (causes outer corners of lips to stretch); 24 , lip press, caused by medial portion of obicularis oris; 25 , lips are parted (slight jaw drop); 26 , lips are parted, and so are teeth; 41 , eyelids are drooping or hanging somewhat. A + symbol means the action is present. Intensity of the action is indicated by letter: A means that it is present to some extent and D that it is very marked indeed. 
this action was only weakly available in the exemplar images. Figure 6 (Experiment 3 ) shows that the individual exemplars for fear were unreliably named. The FACS analysis also suggests that the reason that fear failed to generate caricature effects (Experiments 1 and 2) was because of this relative absence of this distinctive attribute of the expression.

One further slight discrepancy can also be diagnosed from the FACS analysis. A spontaneous anger face usually shows some medial action of the orbicularis oculi, pars palpabrae (AU7). This action raises the lower eyelid slightly to increase glare. AU7 was absent in the anger display.

Did the caricatures increase the FACS ratings of the target expression? In every case, the caricatured image showed key aspects of the expression through the more intense display of the critical muscle actions. This is most clearly evident in the higher ratings of intensity for the critical action systems (i.e., D compared with B or A).

The one apparent exception to this, that of anger, paradoxically confirms the point. Scores for AU5 are rated as being less strong for the caricature than for the composite. Although anger requires orbicularis oculi (AU6), the concomitant actions of the forehead and the lower face interact with this, so that in more intense anger, eye opening is somewhat more constricted.

FACEM measures of facial energy. A small family of just 12 facial measurements appears to be sufficient for people to discriminate the major classes of expression display and clinical differences within them (the facial expression measurement [FACEM] model of Pilowsky, Thornton, \& Stokes, 1985; Thornton \& Pilowsky, 1982). Taxonomies of expression categories have been derived using FACEM (Pilowsky et al., 1985). The following distance measures were taken for each of the composite prototype images (six expressions and neutral): (1) between outer canthi of left and right eyes, (2) mouth width, (3) mouth opening, (4) eyebrow separation, (5) average eye opening/height, (6) canthi to tip of nose, (7) canthi to mouth corner, (8) eyebrow raise, (9) lower lip thickness, and (10) upper lip thickness. Ten of the 12 FACEM measures were used because 2 of the scalar measures around the eye region cannot be consistently delineated in all faces (Benson, in press). ${ }^{4}$

Facial energy, measured as the difference between neutral and target expressions, should be expected to correlate with caricature, inasmuch as FACEM measures are a subset of those used to produce the caricature and are based on the same subtractive manipulation for identical images.

Table 3 shows energy measures taken separately for composite prototype and caricature images in the present study. Net caricature effects for each of the experiments are also shown. Stimulus energy was computed as follows. For each face, the following calculation was performed, using 10 facial measures

$$
\sum_{i=1}^{10}\left|\frac{E_{i}}{N_{i}}-1\right|,
$$

where $N$ is the neutral and $E$ a nonneutral expression. This means that the neutral expression is normalized to zero. The derived number is a description of the relative spatial disparity of combinations of feature measures in one expression over the neutral expression (the facial energy of the expression in structural terms).

There was no significant correlation between (mean) facial energy (columns 1 and 2) and any of the (mean) caricature measures shown in the table. Caricature and prototype energy scores, as expected, correlate highly $[r(4)=.90, p<$ $.001]$, since the FACEM measures were a subset of the measures taken as the basis for the caricature algorithm. This correlation may be taken as further validation for FACEM as a (reduced) measure of expression differences in facial image terms. ${ }^{5}$ More interestingly, the higher order energy score (the caricatured prototype energy difference measure, column 3 ) correlates significantly with the strength of the caricature effect in Experiment 1 [column 4: $r(4)=.74, p<$ .05 , two-tailed], but not with the other caricature scores.

Thus, although it was not the case that faces with the least energy benefit most from caricature, a derived energy measure (the difference between the caricature and the prototype energies) correlates well with target expression ratings in Experiment 1. The greater the caricatured

Table 3

FACEM-Derived Facial Energy Scores and

Net Enhancement (Caricature) Effects Across Experiments

\begin{tabular}{|c|c|c|c|c|c|c|c|}
\hline Expressions & $\begin{array}{l}\text { Energy of } \\
\text { Prototype }\end{array}$ & $\begin{array}{l}\text { Energy of } \\
\text { Caricature }\end{array}$ & $\begin{array}{l}\text { Energy of } \\
\text { Net Effect* }\end{array}$ & $\begin{array}{l}\text { Experiment } 1 \\
\text { Net Rating } \dagger\end{array}$ & $\begin{array}{l}\text { Experiment } 2 \\
\text { Net Rating } \dagger\end{array}$ & $\begin{array}{c}\text { Experiment } 3 \\
\text { Reaction Time }+\end{array}$ & $\begin{array}{l}\text { Experiment } 3 \\
\text { Net Rating } \ddagger\end{array}$ \\
\hline Fear & 0.26 & 0.53 & 1.04 & 0.26 & 0.08 & 0.05 & 0.13 \\
\hline Surprise & 0.52 & 0.94 & 0.81 & 0.16 & 0.20 & 0.00 & -0.05 \\
\hline Sadness & 0.11 & 0.21 & 0.91 & 0.22 & 0.33 & -0.02 & 0.05 \\
\hline Disgust & 0.19 & 0.30 & 0.58 & 0.02 & 0.11 & 0.02 & 0.05 \\
\hline Happiness & 0.39 & 0.77 & 0.97 & 0.20 & 0.21 & -0.02 & 0.06 \\
\hline Anger & 0.18 & 0.30 & 0.67 & 0.16 & 0.28 & 0.47 & 0.28 \\
\hline
\end{tabular}

Note--Energy scores obtained for prototype and caricatured expressions are compared with the net facilitation effects of caricaturing observed in Experiments 1-3. The energy score is a number representing a measure of the strength of each expressive stimulus, relative to a neutral baseline for each of the expression images. *Net stimulus strength (energy) measure for enhanced (C)aricature and (P)rototype stimuli, $(\mathrm{C}-\mathrm{P}) / \mathrm{P}$. ${ }^{\dagger}$ Rating effects, $(\mathrm{C}-\mathrm{P}) / \mathrm{P}$, for mean caricature and mean prototype ratings. $\quad{ }^{\ddagger}$ Reaction time effects, $(\mathrm{C}-\mathrm{P}) / \mathrm{P}$, for mean caricature and mean prototype naming latencies. 
Table 4

Ratings Purity in Experiment 2 Related to Enhancement Effects in Experiments 1 and 3

\begin{tabular}{lccccc}
\hline & $\begin{array}{c}\text { Purity of } \\
\text { Expression }\end{array}$ & $\begin{array}{c}\text { Purity of } \\
\text { Prototype }\end{array}$ & $\begin{array}{c}\text { Caricature } \\
\text { Net Rating* }\end{array}$ & $\begin{array}{c}\text { Experiment 3 } \\
\text { Net Reaction Time† }\end{array}$ & $\begin{array}{c}\text { Experiment 3 } \\
\text { Net Rating* }\end{array}$ \\
\hline Fear & 68.23 & 73.68 & 0.26 & 0.05 & 0.13 \\
Surprise & 66.74 & 80.04 & 0.16 & 0.00 & -0.05 \\
Sadness & 64.09 & 85.15 & 0.22 & -0.02 & 0.05 \\
Disgust & 67.72 & 75.49 & 0.02 & 0.02 & 0.05 \\
Happiness & 73.48 & 91.36 & 0.20 & -0.02 & 0.06 \\
Anger & 58.25 & 74.70 & 0.16 & 0.47 & 0.28 \\
\hline
\end{tabular}

Note-Purity or clarity of observed rating scores. Purity coefficients were calculated separately for prototype and caricatured (enhanced) expressions in Experiment 2, using

$$
\frac{T_{e}-\left(\sum_{i=1}^{6} E_{i}-T_{e}\right)}{\sum_{i=1}^{6} E_{i}},
$$

where $T_{e}$ is the target expression score, and $\sum E$ is the summed expression score. $T_{e}$ is comparable to the value $\sum E_{i}$ in Equation 1 over the range of within-face formalized individuating spatial expression measures. *Rating effects, $(\mathrm{C}-\mathrm{P}) / \mathrm{P}$, for mean caricature and mean prototype ratings. ${ }^{\dagger}$ Reaction time effects, $(\mathrm{C}-\mathrm{P}) / \mathrm{P}$, for mean caricature and mean prototype naming latencies.

energy, the higher the expression rating - where ratings are sought on target expressions only.

Purity: Correlations across the experiments. Although measurable changes in facial energy can predict the caricature effect for Experiment 1, it is clear that, in Experiment 2, the mixedness of some of the expressions played an important role, with nontarget as well as target expressions showing enhancement. Can the rated purity of the expression (Experiment 2) predict naming (Experiment 3)? Table 4 shows two purity measures derived from the means of Experiment 2 (Table 3) in relation to the caricature effect in Experiment 3. The RT measure correlated significantly with the purity caricature measure [column 2 : Spearman's rho, $r(4)=-.943, p<.002]$. Purity of expression as assessed by rating in Experiment 2 predicted speed of naming in Experiment 3.

\section{GENERAL DISCUSSION}

This series of experiments explored the extent to which mathematical enhancement (caricature) of a prototype expression displayed in a face could improve its classification. Positive enhancement increased ratings of goodness of expression, negative ones diminished them. A skeptic might find these results trivial - after all, because we have manipulated face-feature values with respect to a resting (neutral) face, the relevant face actions will have been visibly enhanced. Have we simply tailored a set of "emperor's new clothes"? We believe not. First, the composite prototype faces were rated highly for the expression portrayed (Experiment 1). That is, the photographic image qualities of a given expression, averaged over a number of different individual faces, are sufficient to support reliable and high ratings for that expression when composited in this way. Experiment 3 suggested that, nevertheless, there might be idiosyncratic aspects of the target expression that are not captured by averaging. In other words, not all displays of a target expression may have identical image components. Second, although the source images for the caricatures were derived from images that displayed the relevant emotion, the artificial caricature was rated more highly than the prototype for all expressions. ${ }^{6}$ This, despite the fact that these images had not previously been seen and were unlikely to represent natural facial movements (see Ekman \& Friesen, 1975, 1978). The caricature is judged to be a better example of the category than is the prototype from which it derives. This was not a foregone conclusion. For facial identity, only low levels $(10 \%)$ of caricature generate superportraits (Rhodes, 1996), whereas it is entirely possible that enhancement of expression might be predicted to lead to the perception of a facial image as grimacing or distorted. It is possible, for instance, that further levels of caricature $(+200 \%,+300 \%)$ would move beyond the boundaries of good expression to just such rated judgments. These boundaries are worth exploring: They will provide evidence, not so much for the limits of perceived natural movement, but for the conceptual boundaries of the feature space in which expressions are decoded (perception) and encoded (production).

A similar study to this, but with exemplar rather than composite faces as the basis for caricature (Calder et al., 1997) also reported caricature effects in classifying facial expressions. In Calder et al.'s (1997) studies, enhancement of less than $100 \%$ (typically, 50\%-75\%) was found to generate improved expression identification (matching a facial expression to a label). This suggests that caricature effects based on Brennan's (1985) algorithm are robust across different facial images and different test methods but that the extent of caricature that is optimal may depend on the specific test and stimulus conditions. A further as- 
pect of the robustness of the caricature and image synthesis algorithms is that Calder et al. (1997) report that enhancement from two different baselines can be effective (however, this may be less significant than it might appear, inasmuch as a neutral face is frequently rated as being slightly happy). A similar robust effect of enhancement (relative insensitivity to the baseline measure) was reported in a study of bird caricature (Rhodes \& McLean, 1990).

What do these experiments say about the representational status of facial images of expression? Other studies with morphed facial images from a subset of exemplars from the Ekman series (Calder et al., 1996; de Gelder et al., 1997) have shown that facial expression differences are judged categorically, not continuously. Although categorical structure is certainly implied by these effects, we cannot say that categorical differences between facial expressions are the only ones captured by caricature. As Rhodes and Tremawan (1994) point out, enhancement of category signal can and does occur when the features to be distinguished are simple differences on a continuum (the peak-shift phenomenon; see Thomas, Mood, Morrison, \& Wiertelak, 1991). A dimensional theory of expression (see, e.g., Schlosberg, 1952, 1954) may fit these findings as well as a purely categorical one. It may be more productive to conceive of expressions in terms of neighboring distributions of points in a fairly low dimensional vector space - a conception in which categories are defined by dimensional attributes but cannot be simply reduced to two or three linear dimensions (see Benson, 1995).

If categorical expression structure alone were enhanced by caricature, we would not have expected caricature to affect nontarget as well as target expression classification. In Experiment 2, caricature tended to increase rated values, not only of the target expression, but also of other expression components where these were present in the prototype face to a marked degree. This was particularly clear for fear (surprise rated highly) and disgust (anger rated highly). In Experiment 3, this pattern was again evident. Thus, happiness and sadness (both relatively pure prototypes) (1) were fast to name and (2) showed reliable caricature effects in accuracy. Only anger, of these more mixed emotions, showed a clear caricature effect (both for speed and for accuracy), again in line with the findings of Experiment 2, where anger showed caricature enhancement specific to the target expression. Although enhancement of the purity of anger is still something of a puzzle, the present study supports the more cautious conclusion that, whereas strength of expression is enhanced by caricature, purity may not be to the same extent. The clear relationship (Table 4) between caricature purity, using a measure derived from the ratings of Experiment 2, and expression naming speed in Experiment 3 confirms this picture.

In conclusion, faces portraying a single expression were successfully composited, using computational techniques. Systematic enhancement of feature deviations of these prototyped expressions from those of a neutral face gave rise to more strongly rated expressions. That is, superexpressions can be generated by the application of a sim- ple image enhancement algorithm. However, over the tested range and under our testing conditions, such caricatured images did not reliably speed expression naming, and the pattern of times and errors for naming confirmed that this was probably because caricaturing in this way enhances the strength, not the purity, of each expression.

\section{REFERENCES}

Bartlett, M., Stewart, V., Sejnowski, T., Golomb, B., Larson, J., Hager, J., \& EkMAN, P. (1996). Classifying facial action. In D. Touretski, M. Mozer, \& M. Hasselmo (Eds.), Advances in neural information processing systems (Vol. 8, pp. 823-829). Cambridge, MA: MIT Press.

BENSON, P. J. (1992). Perception and recognition of computer-enhanced facial attributes and abstracted prototypes. Unpublished doctoral thesis, University of St. Andrews.

BENSON, P. J. ( 1994a). Morph transformation of the facial image. Image \& Vision Computing, 12, 691-696.

BENSON, P. J. (1994b). On facial composite image prototyping as a descriptive and diagnostic tool. Journal of Audiovisual Media in Medicine, 17, 27-30.

BENSON, P. J. (1995). Perspectives on face perception: Directing research by exploiting emergent prototypes. In T. Valentine (Ed.), Cognitive and computational aspects of face recognition (pp. 204-224). London: Routledge.

BENSON, P. J. (1998). On the stability of recognising famous faces from photographic-quality caricatures and a conceptualisation of similarity. based processes in long-term memory. Manuscript submitted for publication.

BENSON, P. J. (in press). A means of measuring facial expressions and a method for predicting emotion categories in clinical disorders of affect. Journal of Nonverbal Behavior.

Benson, P. J., \& Katsixitis, M. (1995, August). Production, reproduction, measurement and objective analyses of facial expressions. Paper presented to the 3rd Meeting of the European Society for Philosophy and Psychology. St Catherine's College, Oxford.

Benson, P. J., \& Perrett, D. I. (1991a). Computer averaging and manipulations of faces. In P. Wombell (Ed.), Photovideo: Photography in the age of the computer (pp. 32-38). London: Rivers Oram Press.

Benson, P. J., \& Perrett, D. I. (1991b). Perception and recognition of photographic quality facial caricatures: Implications for the recognition of natural images. European Journal of Cognitive Psychology, 3, $105-135$

Benson, P. J., \& Perrett, D. I. (1991c). Synthesising continuous tone caricatures. Image \& Vision Computing, 9, 123-129.

Benson, P. J., \& Perrett, D. I. (1993). Extracting prototypical facial images from exemplars. Perception, 22, 257-262.

Benson, P. J., \& Perrett, D. I. (1994). Visual processing of facial distinctiveness. Perception, 23, 75-93.

Borod, J. C., St. Clair, J., Koff, E., \& Alpert, A. (1990). Perceiver and poser asymmetries in processing facial emotion. Brain \& Cognition, 13, 167-177.

BrenNan, S. (1985). The caricature generator. Leonardo, 18, 170-178

Calder, A. J., Young, A. W., Perrett, D. I., Etcoff, N. L., \& RowLAND, D. (1996). Categorical perception of morphed facial expressions. Visual Cognition, 3, 81-117.

Calder, A. J., Young, A. W., Rowland, D., \& Perrett, D. I. (1997). Computer-enhanced emotion in facial expressions. Proceedings of the Royal Society of London: Series $B, 264,919-925$.

CAMPBEll, R. (1978). Asymmetries in the interpretation and expression of a facial emotion. Cortex, 14, 327-342.

DARWIN, C. (1965). The expression of the emotions in man and animals. Chicago: University of Chicago Press. (Original work published 1872)

De Gelder, B., Teunisse, J.-P., \& Benson, P. J. (1997). Categorical perception of facial expressions: Categories and their internal structure. Cognition \& Emotion, 11, 1-23.

EKMAN, P. (1982). Emotion in the human face (2nd ed.). Cambridge: Cambridge University Press.

EkmaN, P. (1989). The argument and evidence about universals in facial 
expressions of emotion. In H. Wagner \& A. Manstead (Eds.), Handbook of social psychophysiology (pp. 143-164). Chichester, U.K.: Wiley.

Ekman, P. (1993). Facial expression and emotion. American Psychologist, 48, 384-392.

EKMAN, P. (1994). Strong evidence for universals in facial expressionsA reply to Russell's mistaken critique. Psychological Bulletin, 115, 267-287.

Ekman, P., \& Friesen, W. V. (1975). Unmasking the face: A guide to recognizing emotions from facial clues. Englewood Cliffs, $\mathrm{NJ}$ : PrenticeHall.

EkMAN, P., \& Friesen, W. V. (1978). Facial action coding system: A technique for measurement of facial movement. Palo Alto, CA: Consulting Psychologists Press.

Ekman, P., Friesen, W. V., \& Ellsworth, P. (1972). Emotion in the human face: Guidelines for research and an integration of findings. New York: Pergamon.

Ekman, P., Sorenson, E. R., \& Friesen, W. V. (1969). Pan-cultural elements in facial displays of emotion. Science, 164, 86-88.

Essa, I. A., \& Pentland, A. (1995). Facial expression recognition using visually extracted facial action parameters. In M. Bischel (Ed.), Proceedings of the International Workshop on Automatic Face and Gesture Recognition (pp. 35-40). University of Zurich, Department of Computer Science, Multimedia Laboratory.

Etcoff, N. L., \& MAGEE, J. J. (1992). Categorical perception of facial expressions. Cognition, 44, 227-240.

FridLund, A. J. (1991). Evolution and facial action in reflex, social motive and paralanguage. Biological Psychology, 32, 3-100.

Galton, F. (1907). Inquiries into human faculty and its development (2nd ed.). London: Dent.

IZARD, C. E. (1971). The face of emotion. New York: Appleton-CenturyCrofts.

IZARD, C. E. (1994). Innate and universal facial expressions-Evidence from developmental and cross-cultural research. Psychological Bulletin, 115, 288-299.

Katsikitis, M. (1997). The classification of facial expressions of emotions: A multidimensional-scaling approach. Perception, 26, 613-626.

Mauro, R., \& Kubovy, M. (1992). Caricature and face recognition. Memory \& Cognition, 20, 433-440.

MoRISHIMA, S. (1996). Modelling of facial expression and emotion for human communication system. Displays, 17, 15-25.

NummenmaA, T. (1988). The recognition of pure and blended facial expressions of emotion from still photographs. Scandinavian Journal of Psychology, 29, 33-47.

O'Toole, A. J., Deffenbacher, K. A., Valentin, D., \& Abdi, H. (1994). Structural aspects of face recognition and the other-race effect. Memory \& Cognition, 22, 208-224.

Paramei, G. V., \& Benson, P. J. (1997, July). Subjective dissimilarity of facial expressions is determined by feature topography. Paper presented at the Seventh European Conference on Facial Expression: Measurement and Meaning, Salzburg.

Pilowsky, I., Thornton, M., \& STOKES, B. ( 1985). A microcomputerbased approach to the quantification of facial expressions. Australasian Physical and Engineering Sciences in Medicine, 8, 70-75.

RHODES, G. (1996). Superportraits: Caricatures and recognition. Hove, U.K.: Psychology Press.

Rhodes, G., BRENNAN, S., \& CAREy, S. (1987). Identification and rat- ings of caricatures: Implications for mental representations of faces. Cognitive Psychology, 19, 473-479.

Rhodes, G., Byatt, G., Tremewan, T., Kennedy, A. (1997). Facial distinctiveness and the power of caricatures. Perception, 26, 207-223.

Rhodes, G., \& McLEAN, I. G. (1990). Distinctiveness and expertise effects with homogeneous stimuli: Towards a model of configural encoding. Perception, 19, 773-794.

Rhodes, G., \& TREMAWAN, T. (1994). Understanding face recognition: Caricature effects, inversion and the homogeneity problem. Visual $\mathrm{Cog}$ nition, 1, 275-311.

RusSELL, J. (1980). A circumplex model of affect. Journal of Personality \& Social Psychology, 39, 1161-1178.

RUSSELL, J. (1991). In defence of a prototype approach to emotion concepts. Journal of Personality \& Social Psychology, 60, 37-47.

RuSSELL, J. (1994). Is there universal recognition of emotion from facial expression? A review of the cross-cultural studies. Psychological Bulletin, 118, 102-141.

SCHLOSBERG, H. (1952). The description of facial expressions in terms of two dimensions. Journal of Experimental Psychology, 44, 229-237.

Schlosberg, H. (1954). Three dimensions of emotion. Psychological Review, 61, 81-88.

Thomas, D. R., Mood, K., Morrison, S., \& Wiertelak, E. (1991). Peak shift revisited: A test of alternative interpretations. Journal of Experimental Psychology: Animal Behavior Processes, 17, 130-140.

Thornton, M., \& Pilowsky, I. (1982). Facial expressions can be modelled mathematically. British Journal of Psychiatry, 140, 61-63.

ToMkins, S. S., \& MCCARTER, R. (1964). What and where are the primary affects? Some evidence for a theory. Perceptual \& Motor Skills, 18, 119-158.

Valentin, D., Abi, H., O'Toole, A. J., \& Cottrell, G. W. (1994). Connectionist models of face processing: A survey. Pattern Recognition, 27, 1209-1230.

\section{NOTES}

1. Identification of caricatures has been variously reported as good as or better than veridical images.

2 . The results reported here form part of a more extensive ratings study, the data for which are reserved for separate publication.

3. Multidimensional scaling (MDS) analyses agree with this, having used a variety of paradigms, including expression measurement (Benson \& Katsikitis, 1995), expression identification (Paramei \& Benson, 1997; Russell, 1980; Schlosberg, 1952, 1954), expression matching (Katsikitis, 1997), and expression magnitude (energies in this paper, Table 3 ; Benson, 1995).

4. The magnitude of regions of image-based intersection between the iris and eyelids (upper and lower) is both a binary (discrete) and scalar measure and is not adequately modeled in FACEM.

5. FACEM requires extension to include, at least, discrete terms describing presence or absence of brow and nose furrows, for example (Benson, in press).

6. While disgust was not caricatured reliably in Experiment 1 , it was in Experiment 2.

(Manuscript received March 13, 1997; revision accepted for publication January 19, 1998.) 